\title{
Child oral health promotion experiences in Northern Ireland
}

\author{
Ruth Freeman, Queens University of Belfast
}

It is a curious state of affairs that the children in Northern Ireland (NI) compared with their counterparts in the Republic of Ireland and Great Britain have some of the worst dental health.

Repeated child dental health surveys either within the UK or between health boards in the north and south of Ireland have shown that the NI children and adolescents have greater experience of dental caries.

It has been suggested that the reason for the poor dental health is a lack of awareness on the part of children, adolescents and parents.

However, there is little support for this supposition as parents, children and adolescents in the north have reasonable levels of oral health knowledge. Could the answer lie in the lack of a coordinated approach to oral health promotion - again the answer is no.

The Regional Oral Health Promotion Group has brought together the various stakeholders to develop a coordinated and evidence-based approach for the promotion of oral health in NI. It would seem that another avenue of investigation must be considered if the answer is to be found.

In Belfast, the Department of Health, Social Services and Public Safety's (DHSSPS) policy document, Priorities for Action: 2001-2002, recognised the importance of targeting social need. The document stated that: 'The New Targeting Social Need initiative is designed to address the connection between poverty and unemployment and poor health and social well-being by skewing Government and Departmental resources towards those in greatest need'.

The DHSSPS clearly identified the need to target those 'individuals in the most disadvantaged areas' with regard not only to health inequalities and health care facilities, but also regarding the promotion of health and the prevention of disease.

There was little doubt that dental caries in children and adolescents in NI were associated with social deprivation and disadvantage. However, what was unpalatable was the idea that providing oral health education and oral health promotion for all might sustain rather than reduce oral health inequalities.

The findings from a three-year evaluation of a break-time policy in primary education suggested not only that oral health inequalities existed, but also that these inequalities had been sustained.

Children attending schools classified ${ }^{1}$ as higher socio-economic status (SES) compared with children attending lower SES schools had less experience of dental
Amendment: This leader is an updated version from the original printed version. Professor Ruth Freeman, not Brian Mullally, is the author.

\section{As long as the teeth and mouth are perceived as in someway separate from the rest of the body, then the promotion of oral health will be sidelined.}

caries, greater oral health knowledge and a greater number of healthier snacking opportunities.

Nevertheless, although a differential existed between children who attended participating higher and lower SES schools, the differences in oral health knowledge and snacking behaviours were much reduced. Furthermore equivalent proportions of these children remained caries free in the three years of the study.

This evaluation pointed to the importance of targeting those 'individuals in the most disadvantaged areas' and the necessity to empower children, those entering adolescence and present and future parents with appropriate oral health knowledge and strategies (eg personal skills) for promoting healthier snacking.

The evaluation highlighted the need to negotiate and interact with other stakeholders on a multi-sectorial basis to allow the provision of 'joined-up' health promotion.

The answer to the question of poorer child and adolescent dental health in NI is clear. It is related to social deprivation and disadvantage. If oral health inequalities are to diminish then oral health promotion can no longer be perceived as the 'Cinderella' of health promotion. It must be 'invited to the ball' and form a central strand of health promotion strategies, firmly based within the policy of targeting social need. As long as the teeth and mouth are perceived as in someway separate from the rest of the body, then the promotion of oral health will be sidelined.

Oral health promotion must be centre stage to allow the greatest interaction with others and thus provide 'joined-up' health promotion. Doing so will permit the targeting of those in greatest need and will assist in relegating oral health inequalities to the past.

Lessons for oral health promotion in the wider European context maybe gained from such contemporary experiences in NI.

Socio-economic status determined by the proportion of children entitled to free school meals. 